\title{
Method to Improve the Linearity of Active Commutating Mixers Using Dynamic Current Injection
}

\author{
Mohammad-Mahdi Mohsenpour and Carlos E. Saavedra \\ Department of Electrical and Computer Engineering, Queen's University, Kingston, ON, Canada, K7L 3N6
}

\begin{abstract}
A double balanced (DBM) CMOS mixer providing high linearity is presented in this paper. A cross-coupled pair used in the IF stage of the mixer to dynamically inject current into the to mixer provide a high linearity. The proposed DBM was fabricated using a standard 130-nm CMOS process and was tested on-wafer. The double balanced mixer delivers $10 \mathrm{~dB}$ conversion gain, $9.5 \mathrm{dBm}$ IIP3, and input $P_{1 \mathrm{~dB}}$ of $\mathbf{- 2 . 4} \mathrm{dBm}$. RF bandwidth of the proposed mixer is $6 \mathrm{GHz}$, covering $0.5 \mathrm{GHz}$ to 6.5 GHz with IF bandwidth of $300 \mathrm{MHz}$. RF to IF and LO to IF isolation are also better than $59 \mathrm{~dB}$ in the whole frequency band. The circuit uses an area of $0.015 \mathrm{~mm}^{2}$ excluding bonding pads and draw $4.5 \mathrm{~mW}$ from a $1.2 \mathrm{~V}$ supply.

Index Terms - CMOS Mixer, Current Commutating Mixer, Dynamic Current Injection
\end{abstract}

\section{INTRODUCTION}

A key challenge in the design of the active mixers is providing acceptable linearity, conversion gain, and noise figure (NF) in recent CMOS nodes with reduced power consumption. Due to the trade off between conversion gain, NF, power consumption, and linearity measures $\left(\mathrm{P}_{1 \mathrm{~dB}}\right.$ and IIP3) of the mixer, different methods have been implemented to improve linearity and gain.

The main methods used to improve the performance of the active mixers are current bleeding [1], folded-switching [2][4] and biasing of RF and LO devices separately and with different currents [5]-[7]. Although all these methods lead to better performance, power consumption increase is inevitable. Moreover, these designs often rely on inductors, leading to higher overall size of the mixer and limiting its frequency response.

In this work, a novel dynamic current injection method is proposed to improve the linearity of the mixer while it also makes it possible to increase the conversion gain further. A cross coupled pair is used to dynamically inject current into the mixer, avoiding compression in the output nodes.

\section{Single Balanced Implementation With AUXILIARY PAIR}

The linearity and NF in an active commutating mixer is mostly determined by the overdrive voltage and current draw of the RF stage, which degrades the mixer's conversion gain and voltage overhead. The inverse also holds: if the mixer's gain is increased then its linearity is degraded. Use of external current sources to provide part of commutating current, lets the designer to improve the linearity further.

Figure 1 shows the schematic of the proposed mixer with cross coupled pMOS devices in the IF stage. In the common

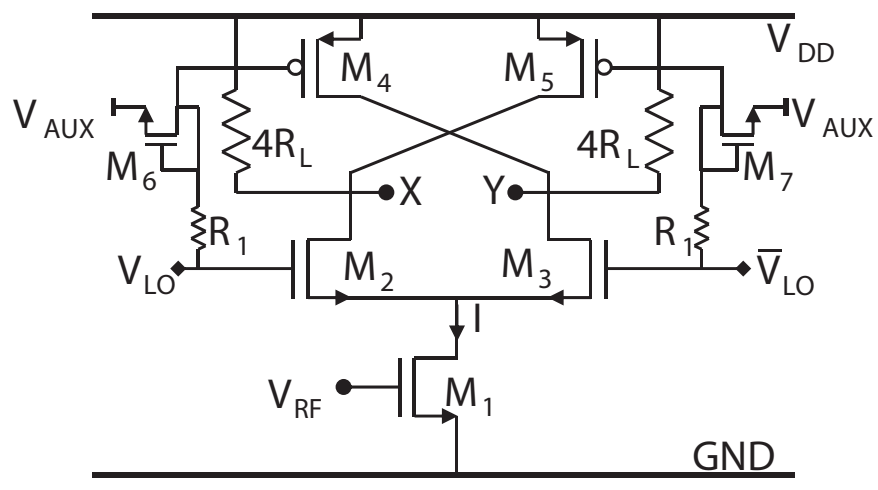

Fig. 1. Schematic of the SBM.

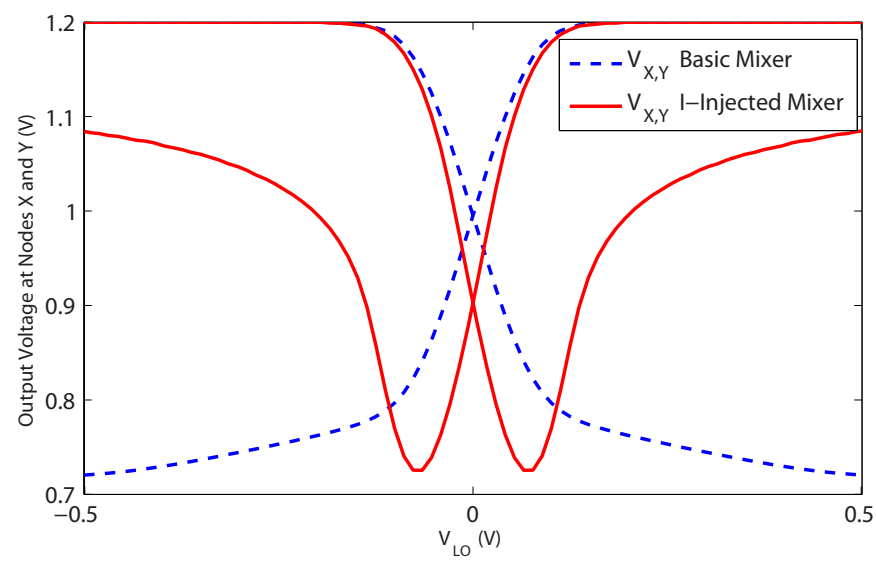

Fig. 2. Output voltage variation with $\mathrm{V}_{L O}$

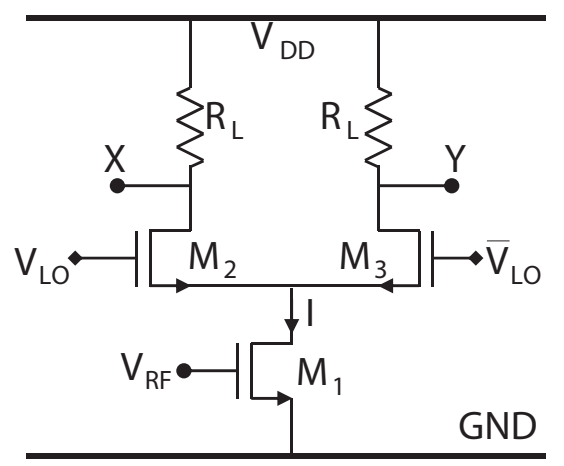

Fig. 3. Schematic of the conventional mixer with static current sources. 


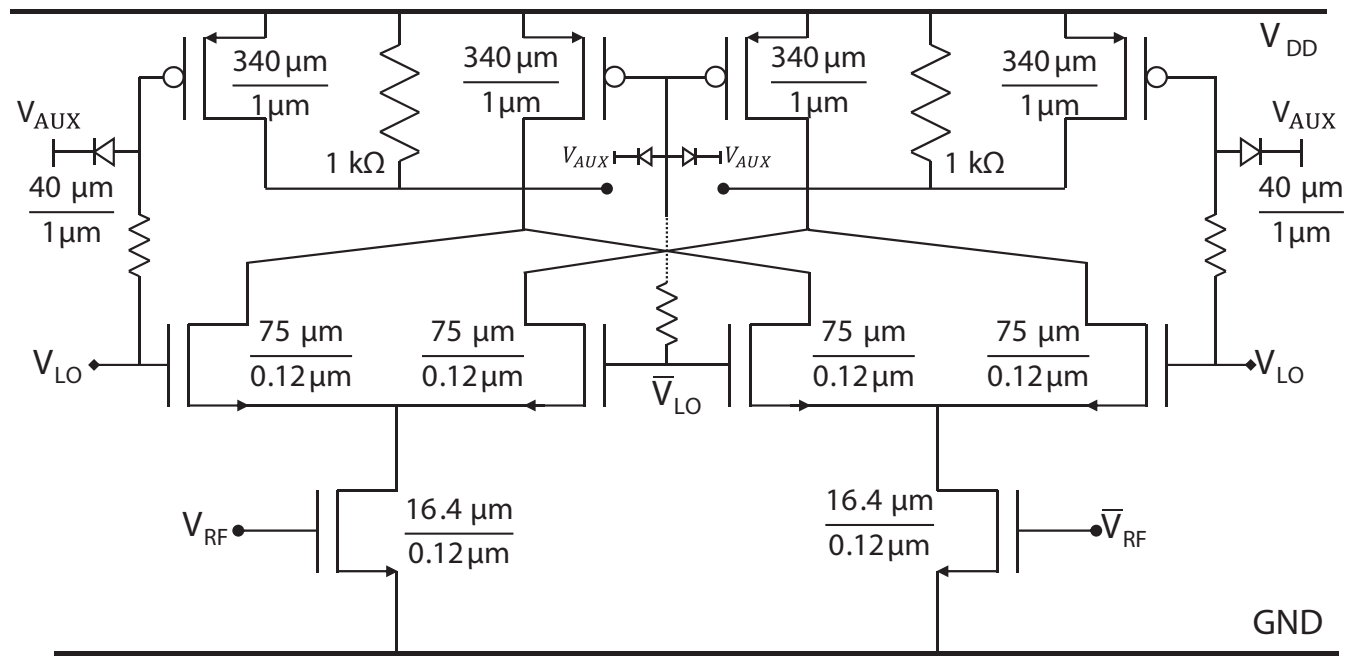

Fig. 4. Schematic of the proposed DBM mixer.

mode state of LO waveforms, currents provided by dynamic current sources are at their minimum, and most of them pass through $4 \mathrm{R}_{L}$. By increasing the LO signal, all the circuit's current goes through the left hand-side eventually, leading to a voltage drop at node $X$. At the same time, the gate voltage of $\mathrm{M}_{4}$ decreases, resulting in a higher current injection to node $\mathrm{X}$ and compensating for the voltage drop in the output. By this method, it is possible to always keep the minimum voltage of nodes $\mathrm{X}$ and $\mathrm{Y}$ above the minimum voltage headroom needed to keep the transistors in saturation and consequently avoiding linearity degradation in the output stage of the mixer.

Figure 2 shows the variation of the output voltages at nodes $\mathrm{X}$ and $\mathrm{Y}$ with instant $\mathrm{LO}$ voltage in a conventional SBM (shown in Fig.3) and the proposed mixer with $4 \mathrm{R}_{L}$ which could lead to $12 \mathrm{~dB}$ higher gain while maintaining the linearity limited to the input. Keeping $\mathrm{M}_{1-3}$ in saturation all the time, ensures limited degradation in the linearity of the mixer while a higher gain is achieved. A pair of diode connected nMOS devices are utilized $\left(\mathrm{M}_{6-7}\right)$ to keep the overdrive voltage of $\mathrm{M}_{4-5}$ and consequently, the injected current limited; avoiding $\mathbf{M}_{4-5}$ from entering into triode region.

To investigate the dynamic current injection method by itself, the RF stage is kept to its most basic implementation. Nonetheless, the proposed method can be integrated with other mixer designs with modified RF stages such as current bleeding and current reuse mixer design techniques due to its completely isolated design from the RF part of the mixer.

For comparison, dynamic current injection method is applied to a basic mixer with $2 \mathrm{~mA}$ current consumption and maximum gain. The SBM with two different set of crosscoupled pairs which provide maximum injection current of $\frac{I}{2}$ and $\frac{3 I}{4}$ was simulated with same device sizes and current consumption.

The SBM with $\frac{3 I}{4}$ maximum current injection, shows 9.2 $\mathrm{dB}$ higher gain compare to the basic mixer while its $\mathrm{P}_{1 \mathrm{~dB}}$ and IIP3 are improved by $4 \mathrm{~dB}$ and $6.25 \mathrm{~dB}$ and become -5.2

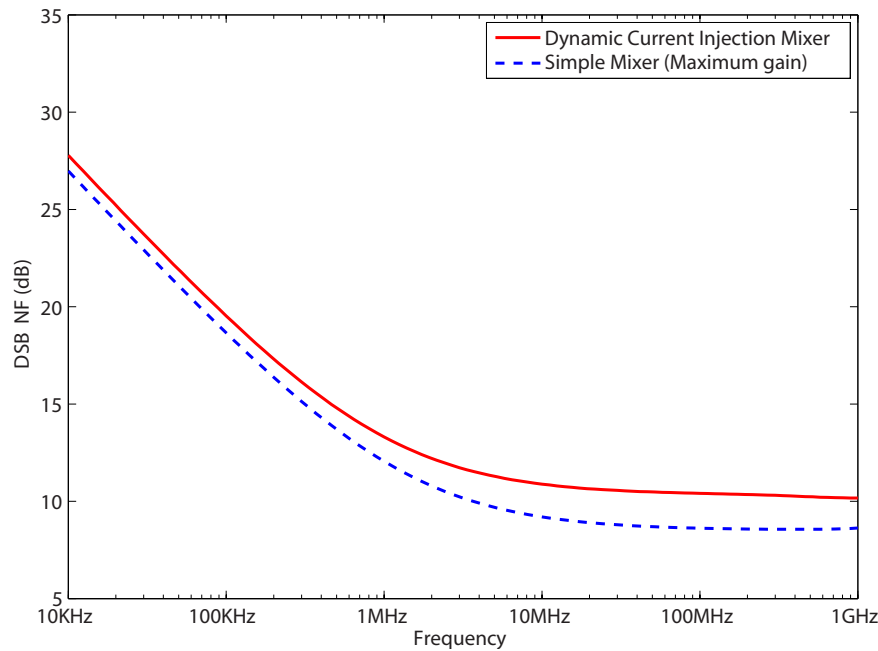

Fig. 5. DSB NF in dynamic current injected SBM mixer and conventional mixer.

$\mathrm{dBm}$ and $6.25 \mathrm{dBm}$, respectively. Noise figure (DSB) is also simulated for both simple mixer with maximum gain and $\frac{3 I}{4}$ maximum current injection mixer. The results are illustrated in Fig. 5 and show low contribution of dynamic current injection to flicker noise of the mixer (only $0.75 \mathrm{~dB}$ higher at $20 \mathrm{KHz}$ IF frequency.) and $2 \mathrm{~dB}$ increase in white noise at $200 \mathrm{MHz}$. The summary of the mixers' performance are also provided in Table I.

The double-balanced version of the proposed mixer is shown in Fig.4, and it is the circuit that was fabricated and whose results are presented in the next section.

\section{Double Balanced Mixer Implementation And MEASUREMENT RESUlts}

In DBM implementation, due to the symmetry of IF branches regarding the LO signal, dynamic current injection 
TABLE I

COMPARISON OF DYNAMIC CURRENT INJECTION MIXER AND CONVENTIONAL MiXER

\begin{tabular}{llccc}
\hline \hline & units & Simple Mixer & $\frac{I}{2}$ Mixer & $\frac{3 I}{4}$ Mixer \\
\hline Conversion Gain & $\mathrm{dB}$ & 2.8 & 6.3 & 12 \\
Input $\mathrm{P}_{1 \mathrm{~dB}}$ & $\mathrm{dBm}$ & -9.26 & -0.4 & -5.2 \\
$\mathrm{IIP3}$ & $\mathrm{dBm}$ & 0 & 11.9 & 6.25 \\
DSB NF $(200 \mathrm{MHz})$ & $\mathrm{dB}$ & 8.5 & 9.5 & 10.25 \\
DSB NF $(20 \mathrm{KHz})$ & $\mathrm{dB}$ & 24.15 & 25.5 & 25 \\
\hline
\end{tabular}

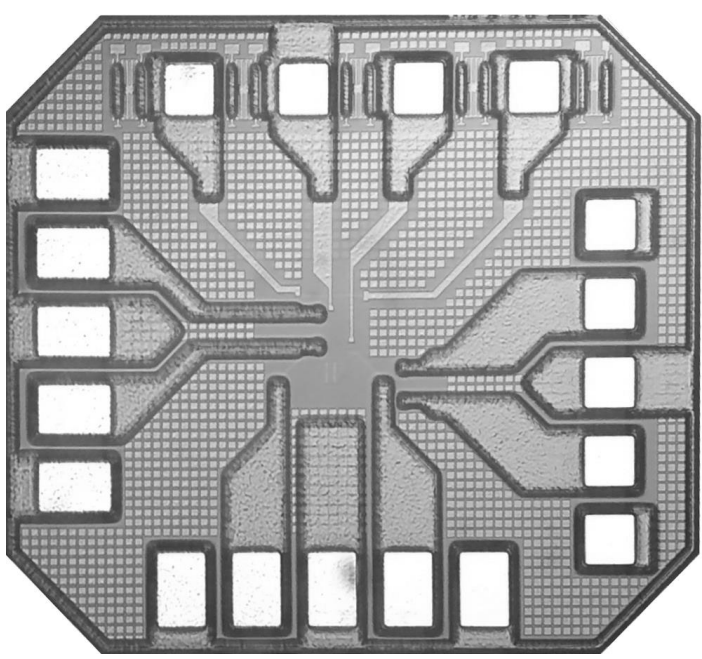

Fig. 6. Microphotograph of the fabricated DBM

devices are also symmetrical in the outputs and in every half period of the LO signal one of them is injecting the current into the output nodes.

The DBM with the dynamic current injection is fabricated using IBM $0.13-\mu \mathrm{m}$ RF CMOS. A microphotograph of the fabricated chip is shown in Fig. 6. The chip core occupies an area of $0.015 \mathrm{~mm}^{2}$, excluding the bonding pads. The circuit consumes $4.5 \mathrm{~mW}$ of dc power from a $1.2 \mathrm{~V}$ supply.

Figure 7 shows the measured and simulated conversion gain results of the mixer in frequency range of $0.5 \mathrm{GHz}$ to $7 \mathrm{GHz}$, for the dynamic current injection mixer, showing a 3-dB gain bandwidth from $0.5 \mathrm{GHz}$ to $6.5 \mathrm{GHz}$. This large bandwidth was expected due to inductor-less design and limiting the modifications to the IF stage of the mixer.

Figure 8 shows conversion gain for different IF frequencies and an IF bandwidth of $300 \mathrm{MHz}$ in this design is achieved. The measurement results, provided for the conversion gain of the mixer, agrees well with the simulation results, with $0.5 \mathrm{~dB}$ degradation, showing a conversion gain of $10.2 \mathrm{~dB}$.

Measured and simulated noise figure are shown in Fig. 9 for $\mathrm{f}_{L O}=2 \mathrm{GHz}$. At IF frequency of $200 \mathrm{MHz}$, NF is $13 \mathrm{~dB}$ while the simulation result shows $12.34 \mathrm{~dB}$. The measured results are provided for frequencies higher than $10 \mathrm{MHz}$ due to calibration limitation of the measurement instruments. Measured and simulated 1-dB compression point are $-2.4 \mathrm{dBm}$ and $-3.4 \mathrm{dBm}$, respectively at $\mathrm{f}_{R F}=2 \mathrm{GHz}$ and $\mathrm{P}_{L O}=4 \mathrm{dBm}$.

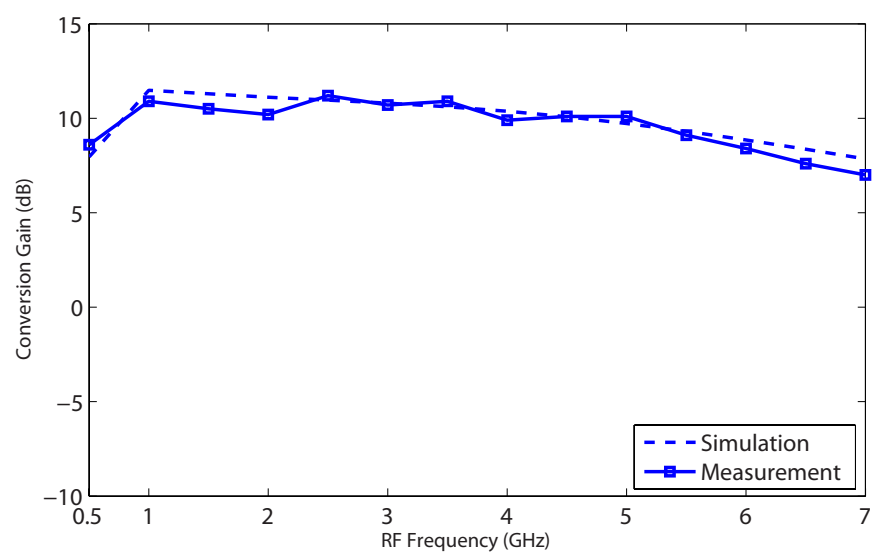

Fig. 7. Measured and simulated conversion gain versus RF frequency.

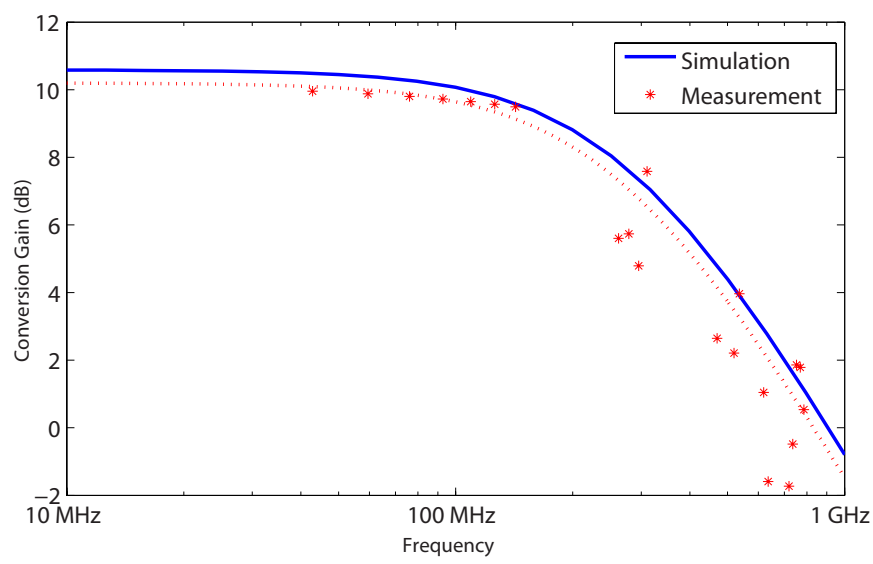

Fig. 8. Measured and simulated conversion gain versus IF frequency.

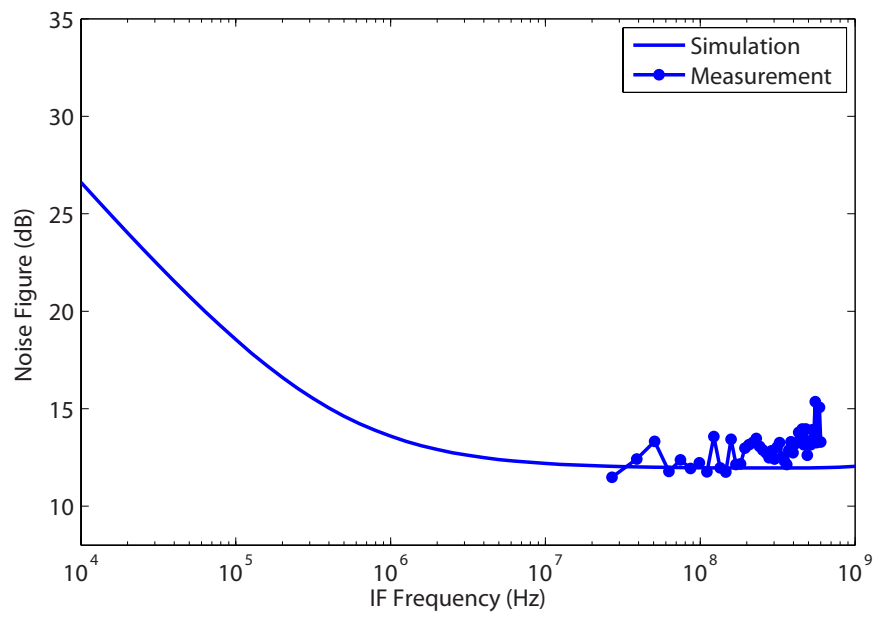

Fig. 9. Measured and simulated DSB Noise Figure versus IF frequency.

$9.52 \mathrm{dBm}$ and $10.56 \mathrm{dBm}$ are respectively, the measured and simulated IIP3 at this frequency. Isolation of the IF to RF and LO signals are also better than $59 \mathrm{~dB}$ in whole frequency band. A summary of the measured results and a comparison with other works is provided in Table II. 
TABLE II

PERFormance Summary AND COMPARISON TABLE

\begin{tabular}{llcccc}
\hline \hline & units & This Work & {$[1]$} & {$[3]$} & {$[6]$} \\
\hline RF Frequency & $\mathrm{GHz}$ & $0.5-6.5$ & $1-5.5$ & $1-10$ & 2.1 \\
IF bandwidth & $\mathrm{GHz}$ & 0.3 & - & $0.1-1$ & - \\
LO power & $\mathrm{dBm}$ & 4 & 0 & 0 & 6 \\
Conversion gain & $\mathrm{dB}$ & 10 & $17.5-13.6$ & $3-8$ & 15 \\
Input $\mathrm{P}_{1 \mathrm{~dB}}$ & $\mathrm{dBm}$ & -2.4 & -10.5 & $-16--12.2$ & - \\
IIP3 & $\mathrm{dBm}$ & 9.52 & 0.84 & $-7--4$ & 15 \\
DSB NF & $\mathrm{dB}$ & 13 & 3.9 & $8.3-12$ & 14 \\
RF-IF isolation & $\mathrm{dB}$ & 64 & - & - & - \\
LO-IF isolation & $\mathrm{dB}$ & 59.76 & - & $>40$ & - \\
DC power & $\mathrm{mW}$ & 4.5 & 34.5 & 8.4 & 8 \\
Chip area & $\mathrm{mm}{ }^{2}$ & 0.015 & 0.315 & 0.28 & - \\
\hline
\end{tabular}

${ }^{*}$ Measured results taken at $2 \mathrm{GHz}$.

\section{CONCLUSION}

A highly linear mixer with boosted gain, based on conventional Gilbert cell is demonstrated in this work. The use of cross-coupled pair to inject dynamic current into the IF stage is the mechanism used to maintain a high linearity while improving the gain. Experimental test on fabricated prototype showed $10 \mathrm{~dB}$ conversion gain alongside $9.5 \mathrm{dBm}$ IIP3.

\section{ACKNOWLEDGMENT}

The authors would like to thank CMC Microsystems, Kingston, Ontario, Canada for chip fabrication arrangements.

\section{REFERENCES}

[1] S. S. Ho and C. E. Saavedra, "A CMOS broadband low-noise mixer with noise cancellation," Microwave Theory and Techniques, IEEE Transactions on, vol. 58, no. 5, pp. 1126-1132, 2010.

[2] H.-K. Chiou, K.-C. Lin, W.-H. Chen, and Y.-Z. Juang, "A 1-V 5-GHz self-bias folded-switch mixer in 90-nm cmos for wlan receiver," Circuits and Systems I: Regular Papers, IEEE Transactions on, vol. 59, no. 6, pp. 1215-1227, 2012.

[3] H. Zijie and K. Mouthaan, "A 1-to 10-GHz RF and wideband IF crosscoupled Gilbert mixer in $0.13 \mu \mathrm{m}$ CMOS," Circuits and Systems II: Express Briefs, IEEE Transactions on, vol. 60, no. 11, pp. 726-730, 2013.

[4] J.-B. Seo, J.-H. Kim, H. Sun, and T.-Y. Yun, "A low-power and high-gain mixer for UWB systems," Microwave and Wireless Components Letters, IEEE, vol. 18, no. 12, pp. 803-805, 2008.

[5] M. Wang and C. E. Saavedra, "Reconfigurable broadband mixer with variable conversion gain," in Microwave Symposium Digest (MTT), 2011 IEEE MTT-S International. IEEE, 2011, pp. 1-4.

[6] M. Mollaalipour and H. Miar-Naimi, "An improved high linearity active CMOS mixer: design and Volterra series analysis," Circuits and Systems I: Regular Papers, IEEE Transactions on, vol. 60, no. 8, pp. 2092-2103, 2013.

[7] K. Choi, D. H. Shin, and C. P. Yue, "A 1.2-v, 5.8-mw, ultra-wideband folded mixer in 0.13- $\mu \mathrm{m}$ CMOS," in Radio Frequency Integrated Circuits (RFIC) Symposium, 2007 IEEE. IEEE, 2007, pp. 489-492. 